\begin{tabular}{|c|c|c|}
\hline Beitr. Ent. & Keltern & ISSN 0005-805X \\
\hline $56(2006) 1$ & S.213-229 & 15.08 .2006 \\
\hline
\end{tabular}

\title{
Some new or poorly known tineids from Central Asia, the Russian Far East and China
}

\section{(Lepidoptera: Tineidae)}

With 18 figures

Reinhard Gaedike

Summary

Results are presented of the examination of tineid material from the Finnish Museum of Natural History, Helsinki (FMNH) and the Institute of Animal Systematics and Ecology, Siberian Zoological Museum Novosibirsk (SZMN). As new species are described Tinea albomaculata from China, Tinea fuscocostalis from Russia, Tinea kasachica from Kazachstan, and Monopis luteocostalis from Russia. The previously unknown male of Tinea semifulvelloides is described. New records for several countries for 15 species are established.

\section{Zusammenfassung}

Es werden die Ergebnisse der Untersuchung von Tineidenmaterial aus dem Finnish Museum of Natural History, Helsinki (FMNH), und aus dem Institute of Animal Systematics and Ecology, Siberian Zoological Museum Novosibirsk (SZMN) vorgelegt. Als neu werden beschrieben Tinea albomaculata aus China, Tinea fuscocostalis aus Russland, Tinea kasachica aus Kasachstan und Monopis luteocostalis aus Russland. Von Tinea semifulvelloides war es möglich, das bisher unbekannte Männchen zu beschreiben. Neufunde für verschiedene Länder wurden für 15 Arten festgestellt.

Keywords

Tineidae, faunistics, taxonomy, four new species, new records, Palaearctic region.

My collegue Lauri Karla from the Finnish Museum of Natural History, Helsinki (FMNH) was so kind as to send me undetermined Tineidae, collected by several Finnish entomologists during recent years in various parts of Russia (Siberia, Buryatia, Far East), in Central Asia, and China. Additionally he sent me material from the Institute of Animal Systematics and Ecology, Siberian Zoological Museum Novosibirsk (SZMN), which $I$ included in the examination. Besides several new records for various countries the material enabled me to describe the previously unknown male of one species, and four new species. 


\section{List of species}

\section{Dryadaula caucasica (ZAGULAJEV, 1970)}

$10^{\star}$, Russia, Buryatia, $54^{\circ} 35^{\prime} \mathrm{N}, 110^{\circ} 48^{\prime} \mathrm{E}$, Bargazin valley, Maisky vill. [age], $500 \mathrm{~m}$, sandy yard, 2.-3.VII.1996, Jalava \& Kullberg (FMNH); 1 9 , Russia, Buryatia, 5340'N, $109^{\circ} 00^{\prime} \mathrm{E}$, Svyatoj Nos, p[e]n[in]s.[ula] Monahovo, $460 \mathrm{~m}$, mixed for.[est] 12.VII.1996, JALAVA \& Kullberg (FMNH); New record for Siberia.

This species was described on the basis of one female from Azerbaidzhan. Later, SATcHKov (1995) published a new record from the Middle Volga region, and described the previously unknown male. As the male genitalia in this genus are extremely asymmetrical and the views in various slides of the same species are very different, it seems useful to publish here another drawing of the male genitalia. Figure 1 shows the uncus-tegumen-vinculum-complex with the very small aedeagus, figure 2 shows the separated valvae.

The longitudinal and transverse strongly sclerotized wrinkles on the last abdominal sternite and the lateromedially notched posterior margin of the two last segments were overlooked in the original description (ZaGulajev 1970, p. 662, fig. 6). Figure 3 shows the corrected drawing of the female genitalia. The female genitalia are very similar to those of D. ussurica.

\section{Hapsifera luridella ZELLER, 1847}

[=pustulatella LUCAS, 1942; = eburnea BUTLER, 1881; = palaestinensis REBEL, 1901; = badiaria TuRATI, 1934; = albicapilla TURATI, 1926; = cyrenaicensis TURATI, 1924; = torulosa Turati, 1919; = baliopsamma MeYruck, 1921; = kerbelella AMsel, 1949; = luridella ssp. susaella AMSEL, 1959; = asiatica AMsex, 1949]

$10^{*}$ Turkmenien: Kugiteng-Gebirge, $5 \mathrm{~km}$ SW Dorf Bazar-Dele, 18.V.1991, leg. V. V. Dubatolov (SZMN)

\section{Montescardia tessulatella (ZELIER, 1846)}

$10^{*}$ S-Ural, Cheljabinsk district, tremel mountain reserve, 900-1200 m, 23.VI.1996, leg. K. Nupponen, J-P. Kartila, J. Jumilainen \& M. Ahola (FMNH); 1 o Russia, Buryatia, $53^{\circ} 40^{\prime} \mathrm{N}, 109^{\circ} 00^{\prime} \mathrm{E}$, Svyatoj Nos p[e]n[in]s.[ula], Monahovo, $460 \mathrm{~m}, 27 .-30 . \mathrm{VI} .1996$, leg. Jalava \& Kullberg (FMNH); $20^{\circ}$, Russia, Buryatia, 5313'N, $109^{\circ} 19^{\prime}$ E, Barguzin valley, $1000 \mathrm{~m}$, Ust-Barguzin Yambui rd., taiga, 13.VII.1996, Jalava \& Kullberg (FMNH)

\section{Montescardia kurenzovi (ZAGULAJEV, 1966)}

Numerous males and females, China, Heilongjiang Yichun, Fenglin reserve, $48^{\circ} 05^{\prime} \mathrm{N}$, $129^{\circ} \mathrm{E}$, ex Fomitopsis pinicola, F rosea, F cajanderi, 2000, leg. Jussi Ikåvalka (FMNH); New record for China. 
Nemaxera betulinella (FABRICIUS, 1787)

[= corticella CURTIS, 1834, nec LINNè, 1758, nec HAWORTH, 1828; = emortuella ZELLER, $1839]$

$10^{\circ}, 1$, Buryatia, $53^{\circ} 40^{\prime} \mathrm{N}, 109^{\circ} 00^{\prime} \mathrm{E}$, Svyatoj Nos p[e]n[in]s.[ula], Monahovo, $460 \mathrm{~m}$, mixed for.[est], 13., 15.7.1996, leg. Jalava \& Kullberg (FMNH)

Nemapogon flavifrons PETERSEN, 1959

[= kabulianus GozMANY, 1960]

10 Kasachstan, Arys, 30.VIII.1992, leg. V. V. Dubatolov \& Logunov (SZMN)

Nemapogon cloacella (HawORTH, 1828)

[= infimella HERRICH - SCHÄFFER, 1851]

10, Altai, Scheljabinskij rajon, Dorf Tscherga, 9.VI.1994, leg. V. K. Zintschenko (SZMN); $2 \sigma^{*}$ Novosibirsk, Akademgorodok, 25., 30.VI.1992, leg. V. V. Dubatolov (SZMN)

Nemapogon wolffiella KaRSHOLT \& SCHMTDT NIELSEN, 1976

[= albipunctella HaWorth, 1828, nec Denis \& SCHIFFERMüLler, 1775]

1 ㅇ, Buryatia, $460 \mathrm{~m}, 53^{\circ} 40^{\prime} \mathrm{N}, 109^{\circ} 00^{\prime} \mathrm{E}$, Svyatoj Nos, Monahovo, 13.VII.1996, leg. Jalava \& Kullberg (FMNH)

Nemapogon inconditella (LUCAs, 1956)

[= buckwelli LuCAs, 1956; = heydeni Petersen, 1957; = thomasi CAPuse, 1975; = hungaricus Capuse, 1968, nec Gozmany, 1960]

1 ㅇ, O-Kasachstan, [village] Glubokoje, 18.-19.VIII.1990, leg. V. K. Zintschenko

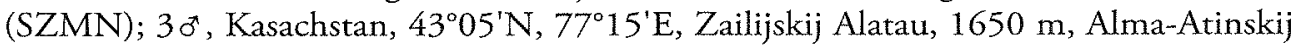
Nat.P., 11., 14.VII.1990, leg. L. Kaila \& K. Miknola (FMNH); $2 \sigma^{\circ}$, Novosibirsk, Akademgorodok, 23.VII.1988, 5.VII.1992, leg. V. V. Dubatolov (SZMN)

Nemapogon robusta GAEDIKE, 2000

1 오 S. Primorje, $43^{\circ} 16^{\prime} \mathrm{N}, 134^{\circ} 08^{\prime} \mathrm{E}$, Lazovskij Res., $180 \mathrm{~m}, 6 .-9.8 .1998$, leg. Jalava, Kullberg \& KaARE (FMNH); First record other than the type series.

\section{Nemapogon gerasimovi ZAGULAJEV, 1961}

$20^{\star}$, NE-China, Heilongjiang dist., Fenglin Nature reserve, $48^{\circ} 05^{\prime} \mathrm{N}, 129^{\circ} 85^{\prime} \mathrm{E}, 200-$ $500 \mathrm{~m}, 12 .-19.6 .2000$, leg. Jussi IKÅVALKA (FMNH); First record from China. 
Nemapogon brandti GAEDIKE, 1986

$1 \sigma^{\star}$, Turkmenien, Kara-Kala, Parchaj, 10.V.1989, leg. et Coll. P. Ivinskis; First record other than the typical series.

Nemapogon variatella (Clemens, 1859)

[= personella PIERCE \& MetcAlfe, 1934; = secalella ZACHER, 1938; = infimella Corbet, 1943, nec HERRICH-SCHÄFFER, 1851]

10 , Novosibirsk, Akademgorodok, 5.VII.1992, leg. V. K. ZinTschenko (SZMN); 1 \%, Ost-Kasachstan, [village] Glubokoje, 1.-2.IX.1990, leg. V. K. Zintschenko (SZMN)

Nemapogon orientalis PETERSEN, 1961

$1 \sigma^{\circ}$, Kasachstan, $43^{\circ} 05^{\prime} \mathrm{N}, 77^{\circ} 15^{\prime} \mathrm{E}$, Zailijskij Alatau, Alma-Atinskij NatP., 21.VI.1990, leg. L. Kaila \& K. Mikkola (FMNH); New record for Kazachstan.

Neurothaumasia fasciata PETERSEN, 1959

Turkmenistan: $10^{*}, 1$ 오 Z-Kopetdag, Firjuza, 8., 20.VII.1991, leg. V. V. Dubatolov (SZMN); 1 ㅇ, Kopetdag, $20 \mathrm{~km}$ O Nochur, Schlucht Karajaltschi, 18.VII.1990, leg. V. V. Dubatolov (SZMN); 1 \&, Kugitang, Airi-vaja, 2150 m, 14.VII.1991, leg. V. V. Dubatolov (SZMN)

Eudarcia (Obesoceras) orbiculodomus (SAKAI \& SAIGUSA, 1999)

$10^{\circ}$, S. Primorje, $43^{\circ} 38^{\prime} \mathrm{N}, 132^{\circ} 33^{\prime} \mathrm{E}$, Ussuriskij Res., $250 \mathrm{~m}, 29 .-31.7 .1998$, leg. Jalava, Kullberg \& KaARe (FMNH); First record other than the typical series, first record for Russian Far East.

\section{Infurcitinea amseli PETERSEN, 1957}

$140^{*}$, Zentral-Kopetdag, $15 \mathrm{~km}$ W Firjuza, Berg Duschak, 7., 8., 9. 10.VII.1990, leg. V. V. Dubatolov (SZMN); New record for Turkmenistan.

Infurcitinea ignicomella (ZELLER, 1852)

[= flavicapilla ZELLER, 1852; = ignicomella HERRICH-SCHÄFFER, 1854]

$50^{x}, 1$ \&, SW-Altai, Katun valley, $10 \mathrm{~km}$ W Katanda, 1200 m, 15.-19.VII.1983, leg. K. Mikkola; H. Hippa \& J. Jalava (FMNH); New record for Siberia.

\section{Infurcitinea grisea PETERSEN, 1973}

$3 \%$, 1 , Turkmenia: Gebirge Kugitang, gegenüber Dorf Bazar-dele, 1720 m, 11 ., 15.V.1991, leg. V. V. Dubatolov \& V. K. Zintschenko (SZMN); New record for Turkmenistan, first record other than the type series. 
Agnatbosia mendicella ([DENIS \& SCHIfFERMüLLER, 1775])

$[=$ propulsatella $($ ReBEL, 1892; = mendicella HüBNER, 1796; = flavimaculella TolL, 1942;

= austriacella AMSEL, 1954]

Numerous males and females, China, Heilongjiang Yichun, Fenglin reserve, $48^{\circ} 05^{\prime} \mathrm{N}$, $129^{\circ} \mathrm{E}$, ex Fomitopsis rosea, 2000, leg. JUSSI IKÅVALKa (FMNH); New record for China.

Myrmecozela lutosella lutosella (EvERSMANN, 1844)

1 \%, Orenburg obl., Orenburgskij zap.[ovednik][=nature reserve], Burtijskij Step, 12.17.06.2001, leg. J. Kullberg \& M. Zalewski (FMNH)

Myrmecozela lutosella gigavea (CHRISTOPH, 1873)

$80^{\prime}$, Kirgizia, 41 $25^{\prime} \mathrm{N}, 76^{\circ} 20^{\prime} \mathrm{E}, 30 \mathrm{~km}$ E Naryn, $2500 \mathrm{~m}, 27$. VII.1990, leg. L. Karla \& K. Mrkkola (FMNH); $10^{*}$, SW-Altai, $15 \mathrm{~km} \mathrm{~S} \mathrm{Katanda,} \mathrm{Bert-kum,} \mathrm{2000-2500} \mathrm{m,}$ 10.-14.VII.1983, leg. K. Mikkola; H. Hippa \& J. Jalava (FMNH)

\section{Myrmecozela dzhongarica ZAGULAJEV, 1971}

20, SW-Altai, Kuragan valley, $15 \mathrm{~km}$ S Katanda, 1000-1800 m, 15.VII.1983, leg. K. Mikkola; H. Hippa \& J. Jalava (FMNH); $60^{\circ}, 2$ ㅇ, Kazachstan, $43^{\circ} 24^{\prime} \mathrm{N}, 75^{\circ} 02^{\prime} \mathrm{E}$, Dzhambulskaja obl., 70 km NNE Frunze, 18., 19.VII.1990, leg. L. KaIr.A \& K. MikrolA (FMNH)

\section{Myrmecozela mongolica PeTERsen, 1965}

Tuva republic: $70^{\circ}, 49,50^{\circ} 44^{\prime} \mathrm{N}, 93^{\circ} 08^{\prime} \mathrm{E}, 1000 \mathrm{~m}$, E Tannu-Ola mts., Irbitei r.[eka]

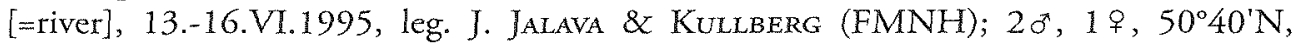
$92^{\circ} 58^{\prime} \mathrm{E}, 750 \mathrm{~m}$, Lake Ubsa-Noor, 15.VI.1995, leg. J. Jalava \& Kullberg (FMNH); New record for Russia.

Ateliotum bungaricellum ZELLER, 1839

[= cypellias MEYRICK, 1937]

2\%, Orenburg obl.[ast], Orenburgskij zap.[ovednik][=nature reserve], Burtijskij Step, 12.-17.06.2001, leg. J. Kullberg \& M. Zalewsiri (FMNH)

Haplotinea insectella (FABRICIUS, 1794)

[= misella ZELLER, 1839; = rusticella HüBNER, 1796, nec HÜBNER, 1813]

10, Tschitinskaja obl., [village] Kyra, 20.VII.1991, leg. V. K. ZINTscheNko (SZMN); $1 \%$, Altai, Tscheljabinskij rajon, [village] Tscherga, 8.VI.1994, leg. V. K. ZINTschenko (SZMN); 1 \%, SW-Altai, Kuragan valley, $15 \mathrm{~km}$ S Katanda, 1200 m, 23.-25.VII.1983, 
leg. K. Mikkola; H. Hippa \& J. Jalava (FMNH); $20^{\circ}$, Burjatia, 543ㅗ' $500 \mathrm{~m}$, Barguzin valley, Maisky vill.[age], 7.VII.1996, leg. J. JaLAVA \& Kullberg

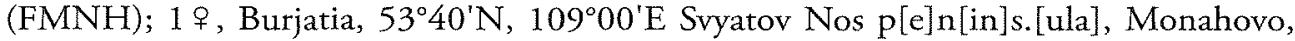
460 m, 13.VII.1996, leg. J. Jalava \& Kullberg (FMNH); 1 , Omskaja obl., 12 km O von Usikkul, 28.-29.VII.1989, leg. S. VAsILENKo (SZMN)

Cepbimallota praetoriella (CHRISTOPH, 1872)

[= uralskella CARADJA, 1920]

10 , Turkmenien, Z-Kopetdag, $15 \mathrm{~km}$ N Firjuza, Berg Duschak, $2150 \mathrm{~m}$, 10.VII.1990, leg. V. V. Dubatolov (SZMN)

Cephimallota colonella (ERSCHOFF, 1874)

[= lignea BUTLER, 1879; = agglutinata MeYrICK, 1931; = colongella ZAGUlAJEv, 1964]

$10^{*}, 1$ ㅇ, Kasachstan, Alma-Ata, 26.IV.1994, leg. V. K. Zintschenko (SZMN); $10^{\star}, 1$ ㅇ, O-Kasachstan, [village] Glubokoje, 2.V.1993, leg. V. K. Zintschenko (SZMN)

\section{Cephimallota tunesiella (ZAGULAJEV, 1966)}

$10^{*}$, Turkmenien, Z-Kopetdag, $15 \mathrm{~km}$ W Firjuza, Berg Duschak, 30.VIII.1988, leg. V. V. Dubatolov (SZMN); 1 q, Kara-Kum, $54 \mathrm{~km}$ N Aschchabad, 31.X.1990, leg. V. V.

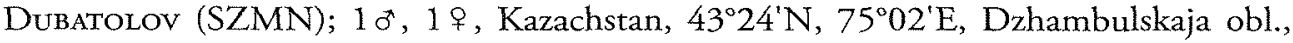
$70 \mathrm{~km}$ NNO Frunze, 15.VIII.1990, leg. K. Mikkola \& J. Kaila (FMNH); New record for Kazachstan.

Cephimallota repetekiella (ZAGULAJEV, 1971)

[= striatella LucAs, 1942, nec DenIs \& SCHIFFERMülLER, 1775; = tunusensis KocaK, $1981]$

2\%, Kara-Kum, 54 km N Aschchabad, 31.X.1990, leg. V. V. Dubatolov (SZMN)

Ceratuncus dzhungaricus ZAGULAJEV, 1971

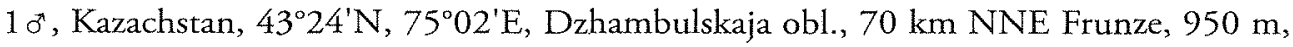
19.VII.1990, leg. J. KaIla \& K. Mikmola (FMNH)

Trichophaga bipartitella (RAGONOT, 1892)

[= amina MeYrIcK, 1925; = desertella MABILLE, 1907]

$40^{\star}, 1$ ㅇ, Russia, Buryatia, $54^{\circ} 35^{\prime} \mathrm{N}, 110^{\circ} 48^{\prime} \mathrm{E}$, Barguzin valley, Maisky vill., $500 \mathrm{~m}$, sandy yard, 2.-3.VII.; 7.VII.1996, JALAVA \& Kullberg (FMNH) 
Trichophaga ziniella ZAGULAJEv, 1960

1 \% , Turkmenien, Zentral-Kopetdag, $15 \mathrm{~km}$ W Firjuza, 7.VII.1990, leg. V. V. Dubatolov (SZMN); $20^{\circ}$, Tuva rep., $50^{\circ} 16^{\prime} \mathrm{N}, 94^{\circ} 54^{\prime} \mathrm{E}, 1250 \mathrm{~m}, 25 \mathrm{~km}$ W Erzin, stony slopes, 7.-

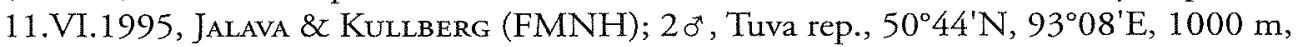
E. Tannu-Ola mts., Irbitei r.[iver], stony steppe slopes, 13.-16.VI.1995, leg. Jalava \& KullberG (FMNH); $30^{\circ}$, Tuva rep., $50^{\circ} 40^{\prime} \mathrm{N}, 92^{\circ} 58^{\prime} \mathrm{E}, 750 \mathrm{~m}$, L. [ake] Ubsa-Noor, 15.VI.1995, leg. J. JALAVA \& KulLBERG (FMNH); $10^{*}$, Tschitinskaja obl.[ast][=region] [village] Kyra, 11.VIII.1991, leg. V. V. Dubatolov (SZMN)

\section{Elatobia fuliginosella (ZELLER, 1846)}

[= martinella WALKeR, 1863; = carbonella Dietz, 1905; = kenteella STAUdinger, 1892; = severella CHRISTOPH, 1888]

$30^{*}$, SW-Altai, Katun valley, $10 \mathrm{~km}$ W Katanda, 1200 m, 15.-19.VII.1993, leg. MikKola; Hippa \& Jalava (FMNH); $10^{7}$, Novosibirsk, Akademgorodok, 29,VI.-3.VII.1984, leg. K. Mikkola \& M. VitTasaari (FMNH); 1 \% , Burjatia, $55^{\circ} 71^{\prime} \mathrm{N}, 111^{\circ} 08^{\prime} \mathrm{E}, 600 \mathrm{~m}$, Barguzin valley, Umjej, 9.VII.1996, leg. Jal.ava \& Kullberg (FMNH); 19 , Burjatia, $53^{\circ} 40^{\prime} \mathrm{N}, 109^{\circ} 00^{\prime} \mathrm{E}, 460 \mathrm{~m}$, Svyatov Nos, pos. Monahovo, 13.VII.1996, leg. JalaVA \& Kullberg (FMNH)

\section{Elatobia kostjuki ZAGULAJEV, 1994}

$10^{\star}, 7$ 오 SW-Altai, Katun valley, $10 \mathrm{~km}$ W Katanda, $1200 \mathrm{~m}, 28 . V I .-5 . V I I ., 15 .-$ 19.VII.1983, leg. Mrkkola; Hippa \& JaLAVA (FMNH); 10 , Burjatia, 700 m, pr. UlanUde, 5 km SW Ulan-Ude, 17.VII.1996, leg. JalaVA \& Kullberg (FMNH); 1 , Burjatia, $54^{\circ} 55^{\prime} \mathrm{N}, 110^{\circ} 48^{\prime} \mathrm{E}, 500 \mathrm{~m}$, Barguzin valley, Maiskij vill.[age], 7.VII.1996, leg. JALAVA \&

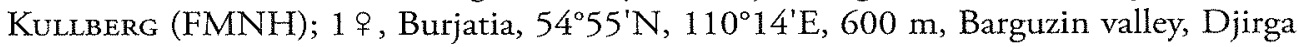
st., 10.VII.1996, leg. Jalava \& KulLBerg (FMNH)

Tineola bisselliella (HuMmeL, 1823)

[= flavifrontella THUNBERG, 1794; = crinella SODOFFSKY, 1830; = destructor STEPHENS, 1834; = lanariella Clemens, 1859; = furciferella ZAGULAJEV, 1954; = anaphecola GozMANY, 1967]

1 은, Tuva rep., $51^{\circ} 43^{\prime} \mathrm{N}, 94^{\circ} 27^{\prime} \mathrm{E}, 700 \mathrm{~m}$, Kyzyl, indoors, 5.-6.VI.1995, leg. JalaVA \& Kullberg (FMNH); 1 đ, O-Kasachstan, Dorf Glubokoje, 5.V.1994, leg. V. K. ZiNTSCHENKO (SZMN)

\section{Tinea dubiella STAINTON, 1859}

[= turicensis MüllER-RuTZ, 1920; = bispinella ZAGUlajev, 1960; = tenerifi ZAGULAJEV, 1966]

10. S-Kasachstan, $35 \mathrm{~km}$ OSO Tschimkent, Georgijevka, 2.IX.1992, leg. V. V. DubaTOLOV \& D. LogunOV (SZMN); New record for Kazachstan. 
Tinea bothniella SVENSSON, 1953

[= unidentella ZAGULAJEV, 1960; = sibiriella ZAGULAJEV, 1960]

1 đ, SW-Altai, Katun valley, $10 \mathrm{~km} \mathrm{~W} \mathrm{Katanda,} 1200 \mathrm{~m}, 15 .-19$. VII.1983, leg. Mrkmola;

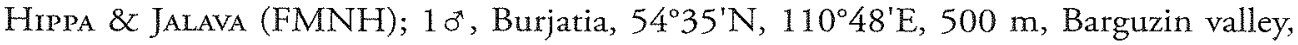
Maijsky vill.[age], 2.-3.VII.1996, leg. JalaVA \& Kullberg (FMNH)

Tinea omichlopis MEYRICK, 1928

[= nonimella ZAGULAJEV, 1955]

1\%, Orenburg obl.[ast, Orenburgskij zap.[ovednik][=nature reserve], Burtinskij Step, 12.-17.06.2001, leg. J. Kullberg \& M. Zalewski (FMNH); $30^{*}$, Novosibirsk, $13 \mathrm{~km} \mathrm{O}$ Berg Karasuk, 11.-13.VIII.1990, leg. V. V. Dubatolov \& L. Ronkay (SZMN); $10^{\star}$, 1 o , Novosibirskaja obl., Karasuk-Steppe, 25.-28.VIII.1982, leg. K. Mrkкola (FMNH); 10, Novosibirsk, Akademgorodok, 10.-20.VI.1983, leg. Miknola; Hrppa \& JaLava (FMNH); 10, O-Kasachstan, d.[erevnja][=village] Glubokoje, 18.VIII.1990, leg. V.

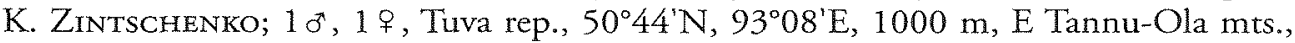
Irbitei r.[iver], 13.-16.VII.1995, leg. Jalava \& Kullberg (FMNH); 10 , Tuva rep., $50^{\circ} 40^{\prime} \mathrm{N}, 92^{\circ} 58^{\prime} \mathrm{E}, 750 \mathrm{~m}$, L.[ake] Ubsa-Noor, 15.VI.1995, leg. Jalava \& KullberG (FMNH); $70^{\circ}$, Burjatia, $54^{\circ} 35^{\prime} \mathrm{N}, 110^{\circ} 48^{\prime} \mathrm{E}, 500 \mathrm{~m}$, Barguzin valley, Maijsky vill.[age], 2.-3., 7.VII.1996, leg. Jalava \& Kullberg (FMNH); $10^{\circ}$, Burjatia, 5501'N, $110^{\circ} 08^{\prime} \mathrm{E}$, $600 \mathrm{~m}$, Barguzin valley, Umhej 9.VII.1996, leg. Jalava \& Kullberg (FMNH)

\section{Tinea columbariella WOCKE, 1877}

[= latro MEYRICK, 1931]

1 ㅇ, Novosibirsk, Akademgorodok, 18.VI.1994, leg. V. K. ZINTSCHENKo (SZMN); 1 đ $^{*}$ Tuva, $5 \mathrm{~km}$ W Kyzyla-Tal des Flusses Ulug-Chem, 15.V.1990, leg. V. V. Dubatolov (SZMN); 1 ㅇ, SW-Altai, Katun valley, $10 \mathrm{~km}$ W Katanda, $1200 \mathrm{~m}, 28$. V.-5.VII.1983, leg. Miknola; Hippa \& Jalava (FMNH); $20^{\circ}$, Burjatia, 5340' N, $109^{\circ} 00^{\prime} \mathrm{E}, 460 \mathrm{~m}$, Svyatov Nos, Monahovo pans. 12., 13.VII.1996, leg. Jalava \& Kullberg (FMNH); $10^{\circ}$, Burjatia, $54^{\circ} 35^{\prime} \mathrm{N}, 110^{\circ} 48^{\prime} \mathrm{E}, 500 \mathrm{~m}$, Barguzin valley, Majsky vill.[age], 7.VII.1996, leg. Jalava \& KullberG (FMNH)

\section{Tinea kasachica sp. nov.}

Terra typica: Kazakhstan: Zailiskiy Alatau

Type: Finnish Museum of Natural History, Helsinki (FMNH)

Imago: Wingspan $13 \mathrm{~mm}$; head, palpi and thorax yellowish, tegulae on the basis overlaid with some darker scales; forewing shining pale yellowish, the costa on the first half with darker scales, three smaller dots with dark scales at $1 / 2,3 / 4$ in the middle of the wing, and at dorsum on the beginning of the ciliae, a narrow line on the basis of the cilia around apex darker, too; hindwing with the same colouration as the forewing. 
Genitalia, of (figs 10-11): Uncus characteristic for the genus, the arms of the gnathos fused in the last fourth; tegumen proximally edged with raised sclerotized narrow band, saccus narrow, as long as the valva; valva broad, parallel, dorsal edge longer than the ventral edge, apically cut, at $1 / 2$ of ventral edge a short, distinct, oblique raised sclerotized band, transtilla very long, the proximal edge of valva from transtilla to the ventral edge crescent-shaped vaulted; aedoeagus a little longer than dorsal edge of valva, outside under apex with some very small teeth, anellus with numerous blunt various-sized teeth.

o: : unknown.

Material examined: $10^{*}$

Holotype, $0^{\circ}$ : “USSR $43^{\circ} 5^{\prime} \mathrm{N} 77^{\circ} 15^{\prime} \mathrm{E}$, Kazakhstan, Zailiskiy Alatau, Alma-Atinskij Nat. [ural] P.[ark];” "1700 m, steppe slope / meadow, 26.6.1990 L. KaIla \& K. MrkKola leg." "Gen[ital]präp.[arat][= genitalia slide] GaEd.[1KE] Nr. 4834;" "Holotypus Tinea kasachica sp. nov. ơ det. R. GAEDIKE 2005."

Remarks: The new species is similar to T. mongolinella Zagulajev, 1972 in the shape of the genitalia, but the aedoeagus and the saccus are shorter, the valva longer and bears a characteristic short, raised sclerotized band at the ventral edge. Superficially mongolinella differs by lack of any darker dots on the forewing.

Derivatio nominis: The new species is named after the location of the type locality.

\section{Tinea semifulvelloides PETERSEN, 1973}

$30^{\star}, 1$ \% , Russia, Kazachstan, $43^{\circ} 5^{\prime} \mathrm{N}, 77^{\circ} 15^{\prime} \mathrm{E}$, Zailijskij Alatau, Alma-Atinskij Nat. [ural] P.[ark], 1700 m, 12.VII.1990, leg. L. Kaila \& K. MikKola (FMNH).

These specimens represent the first records of the species other than the type series. Additionally, the series allows a description of the male genitalia for the first time.

Genitalia of (figs 7-9): Uncus, gnathos and tegumen characteristic for the genus, vinculum at middle with a small triangular sclerotization, saccus long, broadly truncated; valva stout, with a broad basis, costal edge straight, the ventral edge oblique to the blunt top, a sclerotized fold near the ventral edge at $1 / 2$ its length; aedeagus as long as saccus, anellus with a triangular-shaped more or less solid part, and with numerous blunt, various-sized teeth.

\section{Tinea fuscocostalis sp. nov.}

Terra typica: Russia: Chitinskaja region: Sochodinskij Nature Reserve

Type: Institute of Animal Systematics and Ecology, Siberian Zoological Museum Novosibirsk (SZMN).

Imago: Wingspan $17 \mathrm{~mm}$; head light brown, palpi and antennae greyish brown, tegulae light brown, at the basis datk brown; forewing with light yellowish brown ground colour, costa from basis to $1 / 2$ dark brown, to cell at $1 / 3$ vaulted, two small dark brown dots in prolongation of dark brown part of the costa; apex dark brown, wedge-shaped, in lighter colouration, prolonged to the cell, another dark brown dot at dorsum at the beginning of the ciliae, and a very small dot at $1 / 3$ under the cell, the first third of the forewing overlaid with darker brown scales; hindwing brightly shining. 
Genitalia, o (figs 4-6): Uncus, gnathos and tegumen characteristic for the genus, vinculum with a long narrow saccus with rounded tip; valva small, nearly triangular, from transtilla oblique to ventral edge, bordered with narrow sclerotized band, dorsal edge straight from transtilla to blunt rounded apex, ventral edge oblique to apex; aedoeagus as long as saccus, without visible cornuti, anellus with numerous smaller pointed various-sized teeth.

\%: unknown.

Material examined: $1 \sigma^{\circ}$

Holotype, "[Russia: Chitinskaja region], Sochodinskij zap.[ovednik] [= Nature Reserve], r.[eka] [= river] Bukukun, 21.VI.1991, V. V. Dubatolov, V. ZrnTschenko"; "Gen.[ital]präp.[arat] [= genitalia slide] GAED.[IKE] Nr. 4628"; "Holotypus ơ Tinea fuscocostalis sp. nov. det. R. Gaedike 2005".

Remarks: The new species is similar to semifulvelloides, but differs in the markings of the forewings and in the structure of the genitalia. On the forewings of semifulvelloides the two dark brown dots and the dark brown apex are missing. The saccus of semifulvelloides is much longer and broadly truncated, the valva and the anellus are differently shaped.

Derivatio nominis: The new species is named after the colouration of the costa.

Niditinea striolella (MATSUMURA, 1931)

[= piercella Bentinck, 1935; = semidivisa Meyrick, 1934; = distinguenda Petersen, 1957; = ignotella ZAGULAJEV, 1956; = pacifella ZAGULAJEV, 1960]

$10^{*}$, Novosibirsk, Akademgorodok, 25.VI.1992, leg. V. V. Dubatolov (SZMN); $10^{\star}$, Altai, Artyvasch, 19.VII.1992, leg. V. V. Dubatolov (SZMN); 1 \%, SW-Altai, Katun valley, $10 \mathrm{~km}$ W Katanda, $1200 \mathrm{~m}$, 28.VI.-5.VII.1983, leg. Miknola; Hippa \& Jalava $(\mathrm{FMNH}) ; 80^{*}, 2$ 을 Burjatia, $54^{\circ} 35^{\prime} \mathrm{N}, 110^{\circ} 48^{\prime} \mathrm{E}, 500 \mathrm{~m}$, Barguzin valley, Majsky vill.[age], 2.-3., 7.VII.1996, leg. Jalava \& Kullberg (FMNH); $10^{\circ}$, Burjatia, 54 $55^{\circ} \mathrm{N}$, $111^{\circ} 14^{\prime}$ E, Barguzin valley, Djirga st., 10.VII.1996, leg. Jalava \& Kullberg (FMNH); $10^{\circ}$, Burjatia, $55^{\circ} 01^{\prime} \mathrm{N}, 111^{\circ} 14^{\prime} \mathrm{E}$, Barguzin valley, Zugdeli, 9.VII.1996, leg. Jalava \& Kullberg (FMNH); $10^{\circ}$, S. Primorje, $43^{\circ} 36^{\prime} \mathrm{N}, 134^{\circ} 14^{\prime} \mathrm{E}$, Mt Oblatchnaja, $700 \mathrm{~m}$, 3.8.1998, mix. for.[est], leg. Jalava \& Kullberg (FMNH); $50^{*}$, S. Primorje, $43^{\circ} 38^{\prime} \mathrm{N}$, $132^{\circ} 33^{\prime}$ E, Ussuriskij Res., $250 \mathrm{~m}, 29 .-31.7 .1998$, leg. Jalava, Kullberg \& KaAre (FMNH); $10^{\star}$, S. Primorje, $44^{\circ} 02^{\prime} \mathrm{N}, 134^{\circ} 12^{\prime} \mathrm{E}, \mathrm{V}$. Ussuriskij, $550 \mathrm{~m}$, biol. St. Berezovyi sok, 31.7.-5.8.1998, leg. Jalava \& Kuldberg (FMNH); 10 , NE-China, Heilongjiang distr., Fenglin Nature reserve, $48^{\circ} 05^{\prime} \mathrm{N}, 129^{\circ} 85^{\prime} \mathrm{E}, 200-500 \mathrm{~m}, 12 .-19.6 .2000$, leg. Jussi IK̊̊VALKA (FMNH)

Niditinea tugurialis (MEYRICK, 1932)

[= unipunctella ZAgulajev, 1960; = baryspilas MeYRIcK, 1937]

10, S-Kasachstan, 35 km OSO Tschimkent, Georgijevka, 2.IX.1992, leg. V. V. DubaTOLOV \& D. LogunOV (SZMN) 
Monopis spilotella (TENGSTRÖM, 1848)

[= biflavimaculella CLEMENS, 1859]

$2 \sigma^{*}$, NE-China, Heilongjiang dist., Fenglin Nature Reserve, $48^{\circ} 05^{\prime} \mathrm{N}, 129^{\circ} 8^{\prime} \mathrm{E}$, c. $200-$ $500 \mathrm{~m}$, mixed Pinus/deciduous forest, 12.-19.6.2000, leg. Jussi IkÅvalko, 28.6.-10.7. 2000, leg. P. SiHVONEN (FMNH)

\section{Monopis burmanni PETERsen, 1979}

1 ㅇ, Ural, Sverdlovsk, 17.-23.VI.1976, leg. et Coll. V. Zouhar; 1 \%, Burjatia, 5340'N, $109^{\circ} 00^{\prime} \mathrm{E}, 460 \mathrm{~m}$, Svyatrov Nos, Monahovo, 13.VII.1996, leg. Jalava \& Kullberg (FMNH); First records since original description.

Monopis imella (HüBNER, 1810-13)

[= nitidella ZAGULAJEV, 1960]

$30^{\star}$, S-Kasachstan, $2 \mathrm{~km}$ SO Arys', 30., 31.VIII.1992, leg. V. V. Dubatolov (SZMN); 1 o, 1 q , S-Kasachstan, $35 \mathrm{~km}$ OSO Tschimkent, 21.IX.1992, leg. V. V. Dubatolov (SZMN); 2 \%, O-Kasachstan, Dorf Glubokoje, 11.-12.VIII., 2.-3.IX.1990, leg. V. K. ZiNTSCHENKO (SZMN)

\section{Monopis albomaculata sp. nov.}

Terra typica: NE-China: Fenglin Nature Reserve

Type: Finnish Museum of Natural History, Helsinki (FMNH)

Imago: Wingspan $11 \mathrm{~mm}$; Head and palpi yellow, antennae dark brown, on the upper side with pale scales; thorax and forewing dark brown, hyaline area overlaid with a dot with whitish scales, the dot nearly reaches the costa, a second whitish dot on beginning of ciliae at dorsum, along costa and in the apical part of the wing with some very small whitish dots; hindwing light brown.

Genitalia, $\sigma$ (figs 12-13): Uncus cap-shaped, gnathos with broad basis, pointed apically; vinculum in middle broad, towards saccus narrower, saccus at vinculum bifurcate, ends of fork connected with valvae; valva with clearly visible transtilla, ventrally connected with the anellus (it consists of two spoon-shaped parts); shape of valva broad at basis, narrower to blunt apex; first third of costal edge as well of ventral edge more stronger sclerotized as other part of valva; aedoeagus longer than saccus, last third covered with numerous small blunt spines, some longer and pointed spines in vesica.

Genitalia, \&: Unknown.

Material examined: 10

Holotype: "NE-China, Heilongjiang dist.[rict] Fenglin Nature Reserve $48^{\circ} 05^{\prime} \mathrm{N}$, $129^{\circ} 85^{\prime} \mathrm{E}$ c. $200-500 \mathrm{~m}$, mixed pinus/deciduous forest 12.-19.6.2000, Jussi IKåvalko leg."; "Gen.[ital]präp.[arat] [= genitalia slide] GaEd.[IKE] Nr. 5099"; Holotypus o" Monopis albomaculata sp. nov. det. R. Gaedike 2005" 
Remarks: The new species is characterized by the two whitish spots on the forewing. Such whitish markings, but more numerous, and of another size, exist in $M$. henderickxi from Madeira. In the genitalia the shape of the valva and of the anellus are typical for the genus.

Derivatio nominis: The species is named after the colouration of the forewing.

\section{Monopis luteocostalis sp. nov.}

Terra typica: Russia: env. of Irkutsk

Type: Finnish Museum of Natural History, Helsinki (FMNH)

Imago: Wingspan 11-13 mm; head bright yellow, palpi at inner side pale yellow, outside of palpi, antennae, and thorax dark greyish brown; forewing dark greyish brown, costa from $1 / 2$ to apex edged yellow, before apex broader, yellow dot at dorsum, and on the beginning of ciliae; hindwing light grey.

Genitalia, of (figs 14-17); Uncus cap-shaped, apically with small cut, gnathos with broad basis, apically incised; vinculum broad at middle, narrower towards saccus, saccus with proximal prolongation; valva with clearly visible transtilla, ventrally connected with the anellus (it consists of two spoon-shaped parts); costal edge from transtilla to $1 / 2$ with a narrow raised sclerotized edge, the basal edge indented, valva before the middle slightly narrowed, apically broadly round; at ventral edge with many long stronger spines, spines on inner side of valva thin; aedoeagus clearly longer than the valva, vesica with numerous various-sized thorns. Fig. 17 shows the parts of the genitalia in situ.

Genitalia, ㅇ: Unknown.

Material examined: $50^{*}$

Holotype: "USSR, Irkutsk $40 \mathrm{~km} \mathrm{~N}$ steppe slope 1.-3.8.1984 MikKola \& VIITASAARI leg."; "Gen.[ital]präp.[arat] [= genitalia slide] GAEo.[IKE] Nr. 4680"; "Holotypus o" Monopis luteocostalis sp. nov. det. R. GAedrke 2005";

Paratypes: $10^{*}$, with the same dates as the holotype, but: "Gen. [itallpräp. [arat] [= genitalia slide] GAED.[mKE] Nr. 5142"; "Paratypus o Monopis luteocostalis sp. nov. det. R. Gaedrke 2005"; $10^{\circ}$, "Russia Tuva rep.[ublic] $50^{\circ} 16^{\prime} \mathrm{N}, 94^{\circ} 54^{\prime} \mathrm{E}, 1250 \mathrm{~m}$ ca. $25 \mathrm{~km} \mathrm{~W}$ Erzin, steppe/stony slopes 7.-11.6.1995 Jalava \& Kullberg leg."; "Gen.[ital]präp.[arat] [= genitalia slide] GAED. [mKE] Nr. 4801"; "Paratypus o Monopis luteocostalis sp. nov. det. R. GaEDKKe 2005; $10^{*}$, "USSR, SW-Altai Katun valley $10 \mathrm{~km}$ W Katanda, $1200 \mathrm{~m}$, 15.-19.7.1983 Exp. Mikkola, Hippa \& Jalava leg."; "Gen.[ital]präp.[arat] [= genitalia slide] GAED. [IKE] Nr. 5143"; "Paratypus o" Monopis luteocostalis sp. nov. det. R. Gaedike 2005"; $10^{*}$, with the same dates, but: 22.-27.6.1983; "Gen.[ital]präp.[arat] [= genitalia slide] GaEd. [IKE] Nr. 5132"; "Paratypus o Monopis luteocostalis sp. nov. det. R. GaEdike 2005 ". The holotype and three paratypes in the collection of the FMNH, one paratype (genitalia slide Nr. 5132) in the collection of DEI (Deutsches Entomologisches Institut im ZALF, Müncheberg).

Remarks: The new species is distinguishable from other species of the genus by the typical pattern on the forewings. In the male genitalia the proximal prolongation of the saccus is typical.

The new species is named after the colouration of the forewing. 
Monopis monachella (HÜBNER, 1796)

$10^{\circ}$, Burjatia, $53^{\circ} 40^{\prime} \mathrm{N}, 109^{\circ} 00^{\prime} \mathrm{E}, 460 \mathrm{~m}$, Svyatoj Nos, Monahovo, 13.VII.1996, leg. JaLAVA \& Kullberg (FMNH)

Monopis pallidella ZaGuLAJEV, 1955

[= orgidhani GEORGESCU, 1964]

20,1 \%, Novosibirsk, Akademgorodok, 14.-16.VII.1982, leg. K. Mrkkola (FMNH);

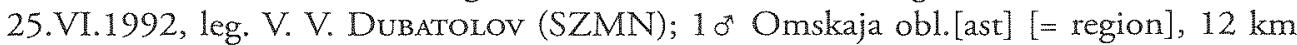
O usil' Kul', 19.-20.VII.1989, leg. VAsilenKo (SZMN); 10, 1 \%, Burjatia, 54.35'N, $110^{\circ} 48^{\prime} \mathrm{E}, 500 \mathrm{~m}$, Barguzin valley, Majsky vill. [age], 7.VII.1996, leg. JaLAva \& KullBerg (FMNH)

The study of the material mentioned above allows a comparison of the female genitalia with the drawing given by PETERSEN (1963: 185, fig. 6) on the basis of a female specimen from NE-Afghanistan. In this drawing the ventral part around the ostium and the dorsal part of the segment were not separated exactly. Figure 18 shows the structure of the female genitalia in more detail.

Monopis christophi PETERSEN, 1957

[ = straminella ZAGULAJEV, 1958]

$10^{\prime \prime}$, Tuva rep., $50^{\circ} 44^{\prime} \mathrm{N}, 93^{\circ} 08^{\prime} \mathrm{E}, 1000 \mathrm{~m}$, E. Tannu-Ola mts., Irbitel r.[iver], 13.16.VI.1995, leg. Jalava \& Kullberg (FMNH); $10^{*}$, Tuva rep., 50 40'N, $92^{\circ} 58^{\prime} \mathrm{E}$, $750 \mathrm{~m}$, L.[ake] Ubsa-Noor, 15.VI.1995, leg.JALAVA \& KullberG (FMNH)

\section{References}

Petersen, G. 1963: 2. Beitrag zur Kenntnis der Tineiden von Afghanistan (Lepidoptera: Tineidae). Beiträge zur Entomologie 13 (1/2): 176-188, 7 figs.

SACHKov [= SATSCHKOV], S. A. 1995: A new and some little-known species of clothes moths (Lepidoptera, Tineidae) from Zhiguli. - Actias; Moskva 2 (1/2): $67-758$ figs.

ZAGULAJEv, A. K. 1970: Dva novych primitivnych vida molej-lichenofagov (Lepidoptera, Tineidae) iz vlazhnych lesov Azerbaidzhana [Two new primitive species of lichenophagous moths (Lepidoptera, Tineidae) from damp forests of Azerbajan]. - Entomologitscheskoje obozrenije 49 (3): 657-664, 6 figs.

Author's address:

Dr. ReINHARd Gaedike

Florusstraße 5

D-53225 Bonn

Germany

email: tinagma@msn.com 

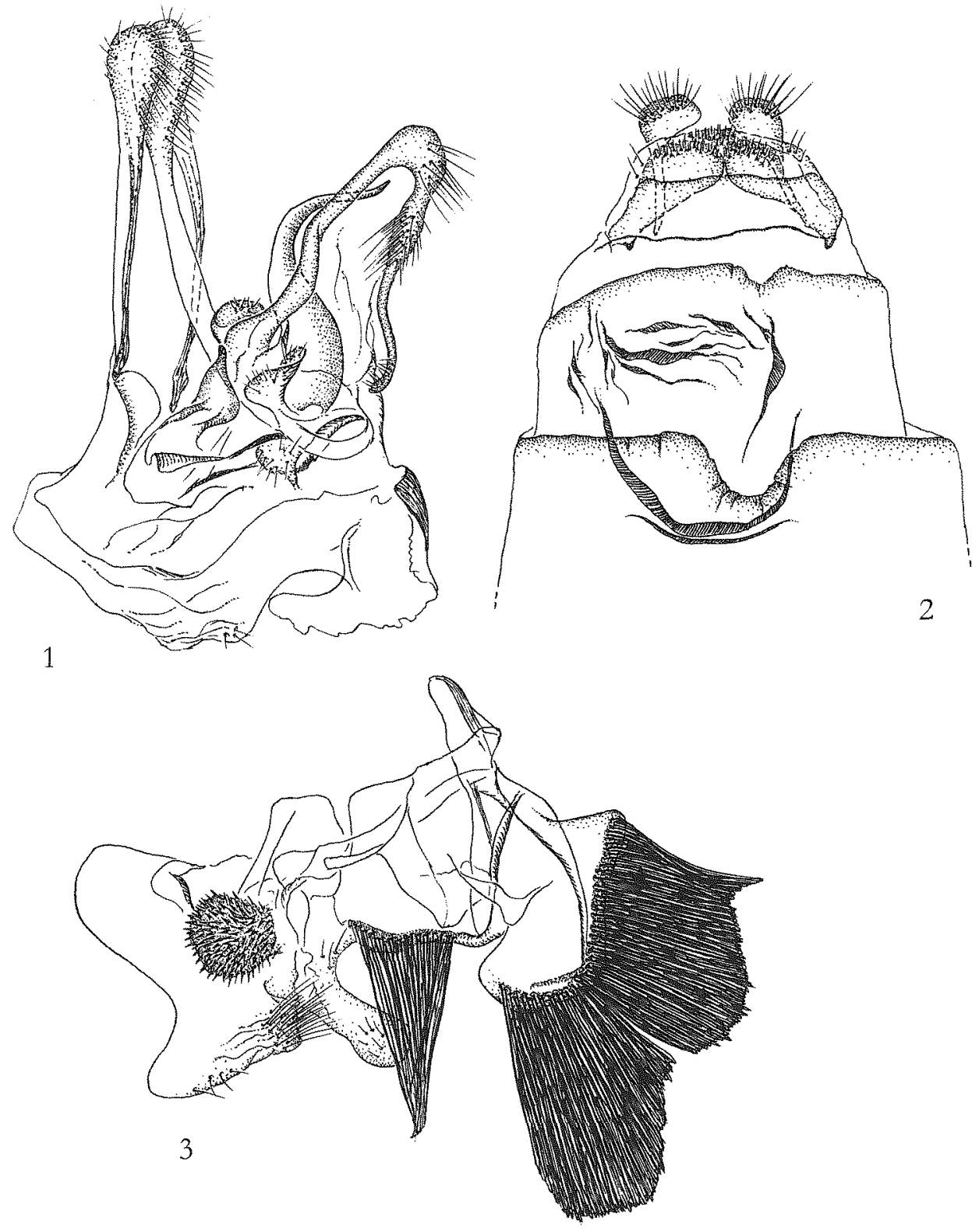

Figs 1-3: Dryadaula caucasica: 1-2, ơ genitalia (1: uncus-tegumen-complex with aedoeagus; 2: valvae), 3 , ㅇ genitalia. 


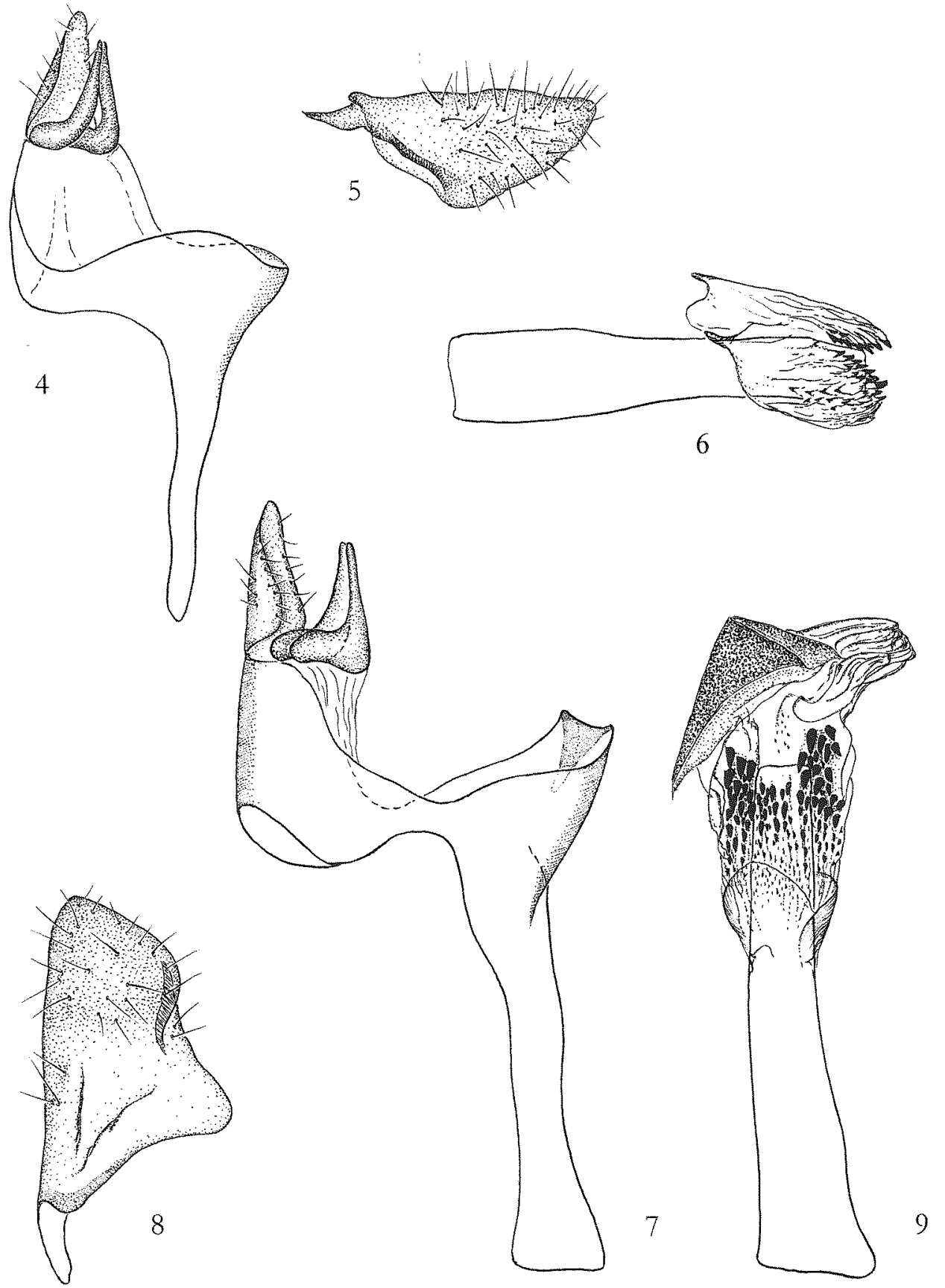

Figs 4-9: ơ genitalia: 4-6. Tinea fuscocostalis (4: uncus-tegumen; 5: valva; 6: aedoeagus); 7-9. Tinea semifulvelloides (7: uncus-tegumen; 8: valva; 9: aedoeagus) 

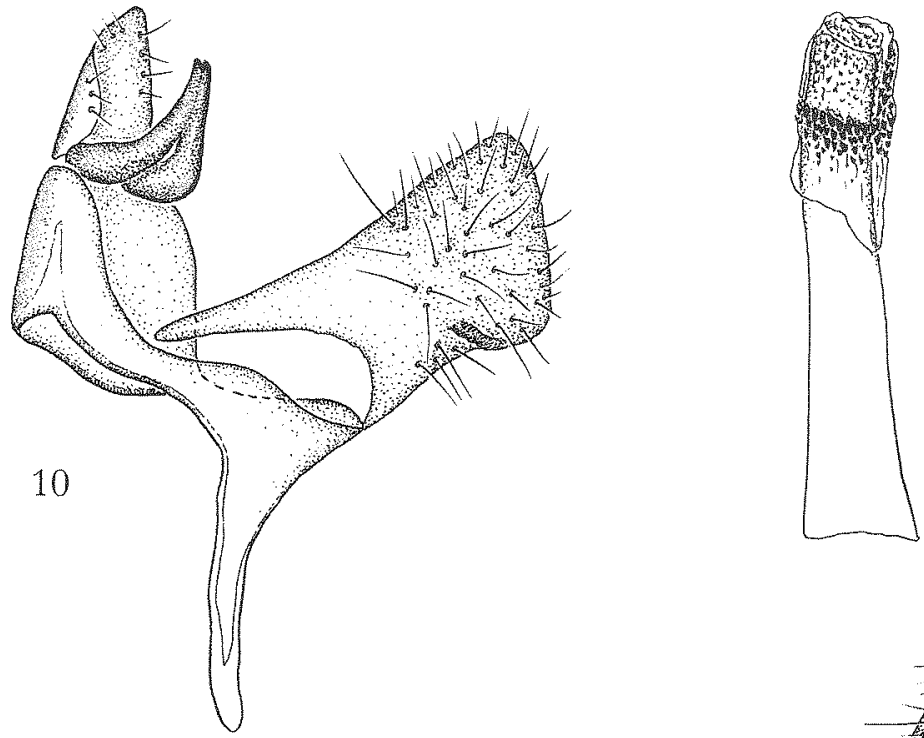

11
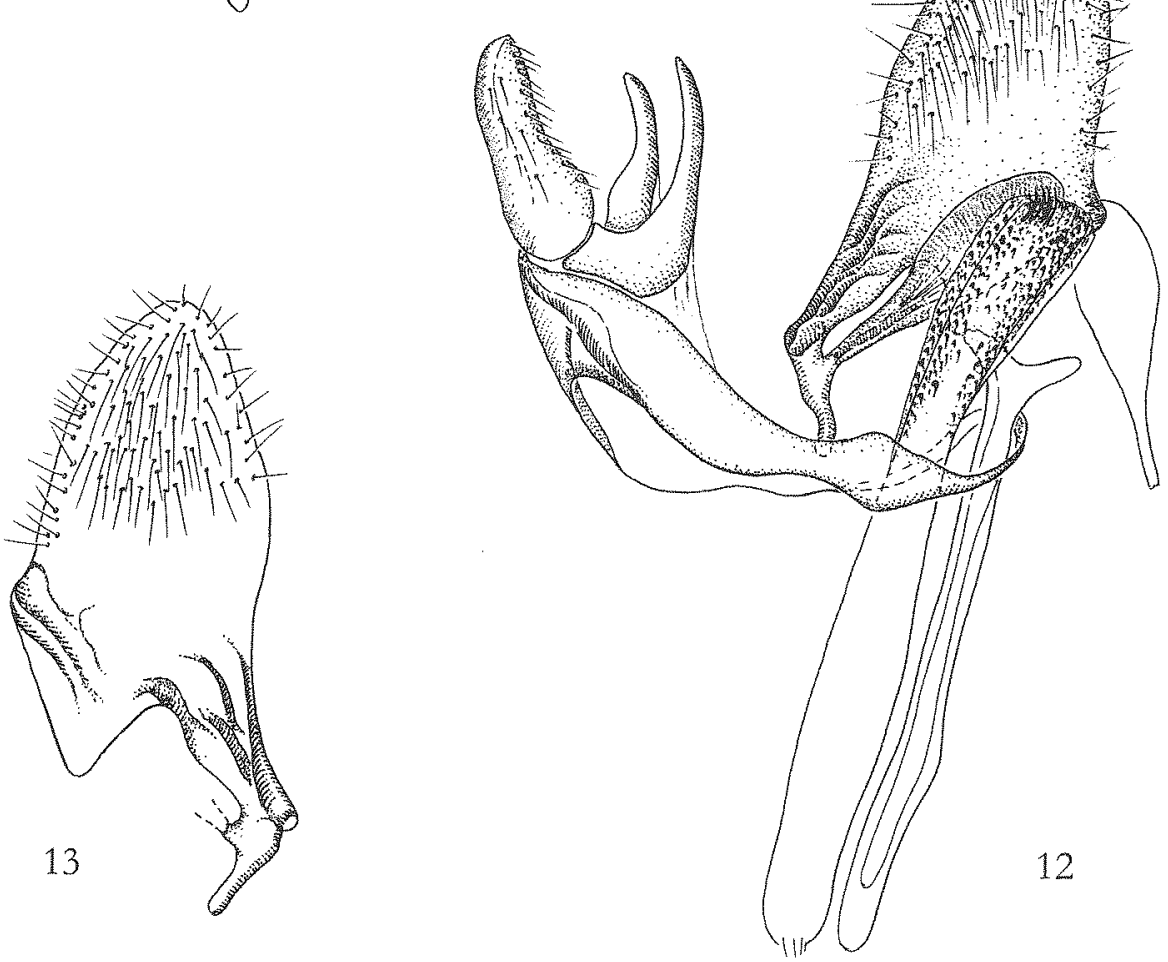

Figs 10-13: $\delta^{*}$ genitalia: 10-11. Tinea kasachica, left valva removed, aedoeagus separated; 12-13. Monopis albomaculata, left valva separated 


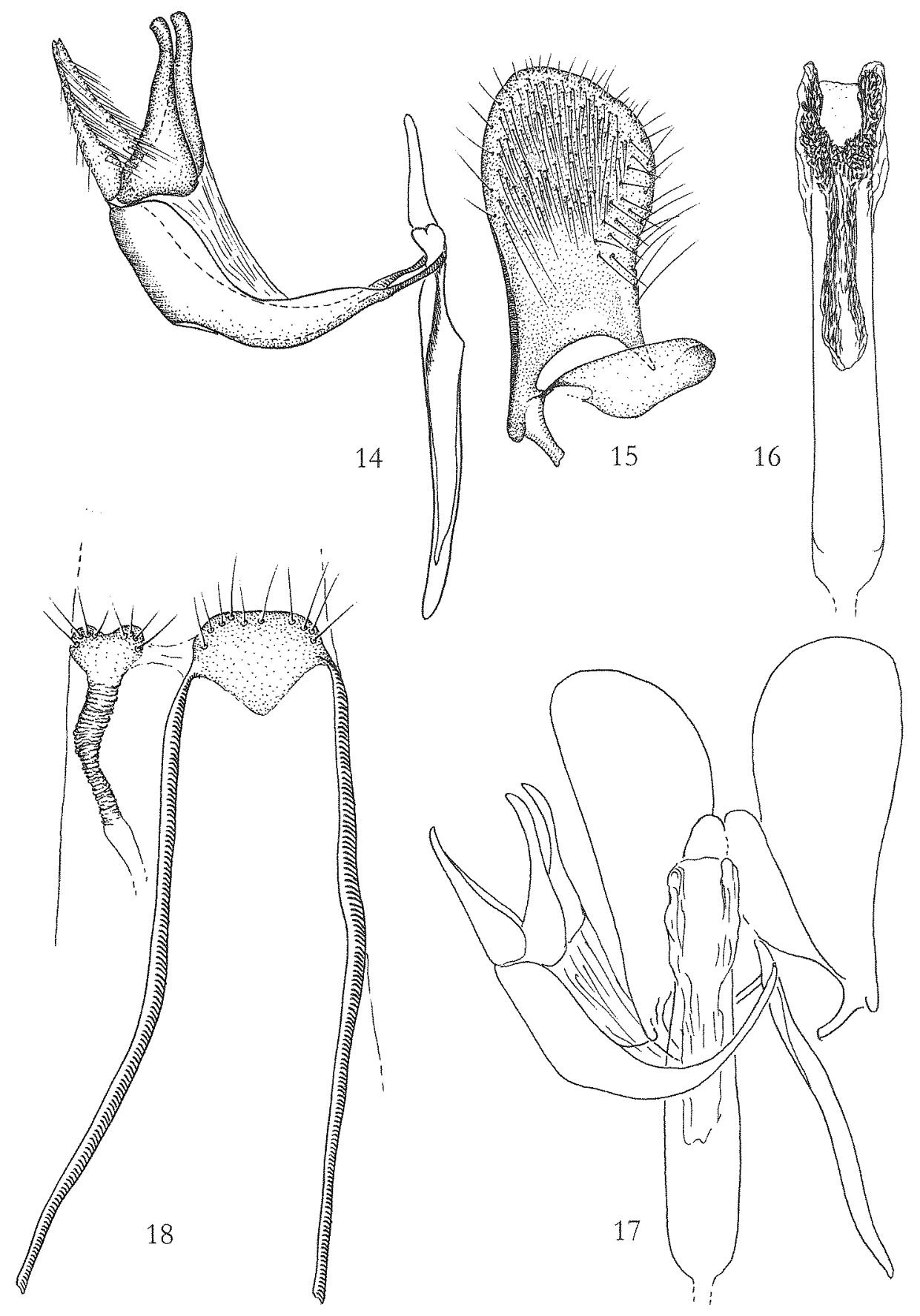

Figs 14-17: Monopis luteocostalis, ơ genitalia (14: uncus-tegumen, saccus; 15: valva; 16: aedoeagus; 17: the whole genital apparatus in situ); Fig. 18: Monopis pallidella, 우 genitalia. 